\title{
Severe hydroxymethylbilane synthase deficiency causes depression-like behavior and mitochondrial dysfunction in a mouse model of homozygous dominant acute intermittent porphyria
}

Stefanie Berger ${ }^{1}$, Miranda Stattmann ${ }^{1}$, Ana Cicvaric ${ }^{1}$, Francisco J. Monje ${ }^{1}$, Pierluca Coiro ${ }^{1}$, Matej Hotka', Gerda Ricken², Johannes Hainfellner ${ }^{2}$, Susanne Greber-Platzer ${ }^{3}$, Makiko Yasuda ${ }^{4}$, Robert J. Desnick ${ }^{4}$ and Daniela D. Pollak ${ }^{1 *}$

\begin{abstract}
Acute intermittent porphyria (AIP) is an autosomal dominant inborn error of heme biosynthesis due to a pathogenic mutation in the Hmbs gene, resulting in half-normal activity of hydroxymethylbilane synthase. Factors that induce hepatic heme biosynthesis induce episodic attacks in heterozygous patients. The clinical presentation of acute attacks involves the signature neurovisceral pain and may include psychiatric symptoms. Here we used a knock-in mouse line that is biallelic for the Hmbs c.500G > A (p.R167Q) mutation with 5\% of normal hydroxymethylbilane synthase activity to unravel the consequences of severe HMBS deficiency on affective behavior and brain physiology. Hmbs knock-in mice (KI mice) model the rare homozygous dominant form of AIP and were used as tool to elucidate the hitherto unknown pathophysiology of the behavioral manifestations of the disease and its neural underpinnings. Extensive behavioral analyses revealed a selective depression-like phenotype in Hmbs Kl mice; transcriptomic and immunohistochemical analyses demonstrated aberrant myelination. The uncovered compromised mitochondrial function in the hippocampus of knock-in mice and its ensuing neurogenic and neuroplastic deficits lead us to propose a mechanistic role for disrupted mitochondrial energy production in the pathogenesis of the behavioral consequences of severe HMBS deficiency and its neuropathological sequelae in the brain.
\end{abstract}

Keywords: Hippocampus, Hydroxymethylbilane synthase, Mitochondria, Mouse behavior, Porphyria

\footnotetext{
* Correspondence: daniela.pollak@meduniwien.ac.at

'Department of Neurophysiology and Neuropharmacology, Center of

Physiology and Pharmacology, Medical University of Vienna,

Schwarzspanierstrasse, 17, A-1090 Vienna, Austria

Full list of author information is available at the end of the article
}

C C The Author(s). 2020 Open Access This article is licensed under a Creative Commons Attribution 4.0 International License, which permits use, sharing, adaptation, distribution and reproduction in any medium or format, as long as you give appropriate credit to the original author(s) and the source, provide a link to the Creative Commons licence, and indicate if changes were made. The images or other third party material in this article are included in the article's Creative Commons licence, unless indicated otherwise in a credit line to the material. If material is not included in the article's Creative Commons licence and your intended use is not permitted by statutory regulation or exceeds the permitted use, you will need to obtain permission directly from the copyright holder. To view a copy of this licence, visit http://creativecommons.org/licenses/by/4.0/. The Creative Commons Public Domain Dedication waiver (http://creativecommons.org/publicdomain/zero/1.0/) applies to the data made available in this article, unless otherwise stated in a credit line to the data. 


\section{Introduction}

Acute intermittent porphyria (AIP), an autosomal dominant inborn error of heme biosynthesis, is due to pathogenic mutations in the hydroxymethylbilane synthase (Hmbs) gene which reduce the activity of its encoded enzyme (HMBS or porphobilinogen deaminase) by approximately $50 \%[1,2]$. AIP heterozygotes are at risk of having life-threatening acute neurovisceral attacks that may be accompanied by psychiatric manifestations [37]. These episodic attacks are triggered by endogenous (e.g. hormones) or exogenous (e.g. drugs) precipitating factors that induce the hepatic expression of $\delta$ aminolevulinic acid synthase 1 , the first and rate-limiting enzyme of heme biosynthesis [8-11] resulting in the massive accumulation of the putatively neurotoxic porphyrin precursors, $\delta$-aminolevulinic acid (ALA) and porphobilinogen (PBG) in the liver plasma and urine.

Patients with the rare homozygous dominant form of AIP (HD-AIP) carry biallelic mutations in the Hmbs gene and have constitutively elevated levels of urinary ALA and PBG due to a 95\% decrease in HMBS activity [12-14]. The clinical presentation of HD-AIP is dominated by neurological impairments and psychomotor retardation [13], which are present without exposure to precipitating porphyrinogenic factors.

An AIP mouse model with 30\% of normal HMBS activity (designated T1/T2 mice) was generated over 20 years ago and used as valuable tool for the investigation of the pathogenesis of AIP and exploration of novel therapeutic strategies for the disease [15-17]. As the clinical and biochemical phenotype in these animals is only apparent after induction with a porphyrinogenic agent, namely phenobarbital [18] which has strong sedative effects, the possibilities for behavioral examinations in this model have been limited.

Recently, a mouse strain carrying a c.500G > A knockin (KI) mutation in the Hmbs gene (p.Arg167Glu; R167Q) was generated, with KI mice displaying striking clinical reminiscence of HD-AIP patients [13]. KI mice with only $\sim 5 \%$ residual murine HMBS activity, have inherently augmented plasma and urine levels of ALA and PBG without induction by a porphyrinogenic drug. These mice also have markedly elevated ALA and PBG concentrations in the cerebral spinal fluid and brain and present with aberrant motor development and defective myelination [13].

Here we hypothesized that characterization of this mouse line and associated molecular underpinnings bears the potential to reveal the consequences of severe HMBS deficiency on behavioral traits and to uncover the underlying pathomechanistic principles. To define the molecular signature paralleling the behavioral phenotype of KI mice we conducted transcriptomic analysis using RNAseq with subsequent immunohistochemical corroboration. We then pursued the possibility that a mitochondrial energy deficit could underlie the observed phenotypic and molecular derangements and defined its consequences for adult hippocampal neurogenesis and synaptic plasticity.

\section{Materials and methods}

Aim, design and setting of the study

The aim of this study was to examine the consequences of severe HMBS deficiency on affective behavior and to examine the underlying pathophysiological principles in a mouse model of homozygous AIP. KI mice harboring a point mutation in the HMBS gene were compared to WT littermate controls in behavioral, molecular and biochemical studies.

\section{Animals}

Heterozygous breeding couples of the R167Q mouse line [13] were generously provided by Makiko Yasuda, MD, PhD, Icahn School of Medicine at Mount Sinai, NY, USA. For all studies, adult homozygous knock-in mutants (KI; p.Arg167Glu; R167Q mice) were compared to sex- and age-matched wildtype (WT) littermate controls.

Animals were single housed under standard conditions in a colony room with a temperature of $22 \pm 1^{\circ} \mathrm{C}$, humidity of $50 \%, \mathrm{CO}_{2}$ controlled to $500-600 \mathrm{ppm}, 12: 12 \mathrm{~h}$ light/dark cycle and food and water available ad libitum unless stated otherwise. The light intensity amounted to 10-20 lx inside the home cages.

For all behavioral, cellular and molecular analyses adult animals (8-12 weeks) were used.

\section{Behavioral analyses}

Behavioral characterization of KI and WT controls consisted of the following tests:

\section{Home cage activity}

Daily home cage activity patterns were tracked and recorded using the PhenoTyper system (Noldus, Wageningen, Netherlands). Mice were placed into the PhenoTyper arena $(30 \times 30 \mathrm{~cm})$ and continuously monitored for $24 \mathrm{~h}$. The EthoVision software (Noldus, Wageningen, Netherlands) was used to determine and quantify relevant behavioral parameters, including total mobility, distance covered and velocity.

\section{Sucrose preference test (SPT)}

The SPT assessing anhedonic behavior was adapted from a standard protocol [19-21]. Briefly, adult, single -housed mice were water and food-deprived for $18 \mathrm{~h}$ before the 2 days habituation period to $2 \%$ sucrose (Sigma-Aldrich, St. Louis, USA) solution. During the $3 \mathrm{~h}$ testing period, mice were given the choice to drink from either of two identical bottles, one filled 
with $2 \%$ sucrose solution, the other with regular tap water. The weight of the bottles before and after testing was determined and used for the calculation of the percentage of sucrose preference relative to the total liquid consumption.

\section{Novelty suppressed feeding (NSF)}

The NSF test was conducted as previously described [22, 23]. Briefly, mice were weighed and food deprived $24 \mathrm{~h}$ prior to behavioral testing. A single food pellet (fixed to a circular paper) was placed into the center of a brightly lit $(800 \mathrm{~lx})$ arena $(30 \times 50 \mathrm{~cm})$. The latency to feed on the pellet was recorded (15 min max time) for each mouse and used as a parameter for the indication of depression-related anxiety.

\section{Light/dark box (LD)}

The LD test was conducted to measure anxiety-like behavior according to a standardized protocol [24] using the Activity Monitor (MedAssociates Inc., St. Albans, VT, USA). Illumination in the light and dark compartment was maintained constant at $200 \mathrm{~lx}$ and $5 \mathrm{~lx}$, respectively. At the beginning of the test, animals were placed into the dark box and allowed to freely explore the arena for $10 \mathrm{~min}$. The amount of time an animal spent in the light area and the transition between the zones was recorded.

\section{Elevated plus maze (EPM)}

Anxiety-like behavior in the EPM was assessed as previously described [24]. Briefly, the plus maze, consisting of two open and two closed arms, was elevated $65 \mathrm{~cm}$ above the floor. Lighting in the open arms amounted to $100 \mathrm{~lx}$ and in the closed arms to $30 \mathrm{~lx}$. At the onset of the $5 \mathrm{~min}$ trial, mice were placed in the center of the maze facing the left open arm. The time the animal spent in the open versus closed arms was recorded by the software Videotrack ${ }^{\circ}$ (Viewpoint, Champagne au Mont d'Or, France) and used as a parameter for the indication of anxiety-related behavior.

\section{Tail suspension test (TST)}

In the TST, behavioral despair was evaluated according to a standard protocol [25]. In short, mice were suspended by their tail in a strain gauge position using a commercial cubicle TST system (MedAssociates Inc., St. Albans, VT, USA). A monitoring system recorded the amount of immobile and mobile time for each animal during the 6 min testing period. The duration of immobility was considered to be an indicator of behavioral despair. SSRI responsivity was tested by acute administration (intraperiteoneal (i.p.)) of Escitalopram (SigmaAldrich, St. Louis, USA) $(10 \mathrm{mg} / \mathrm{kg}$ in $0.9 \% \mathrm{NaCl}) 30 \mathrm{~min}$ prior to the TST [26].

\section{Adult hippocampal neurogenesis}

Progenitor cell proliferation and survival of newborn cells were determined based upon published 5bromodeoxyuridine (BrdU) injection protocols [19, 24]. For the evaluation of progenitor cell proliferation mice were administrated $10 \mu \mathrm{l} / \mathrm{g}$ body weight of $50 \mathrm{mg} / \mathrm{kg}$ BrdU (Sigma-Aldrich, St. Louis, USA) dissolved in 0.9\% $\mathrm{NaCl}$ (i.p.) 5 times in $2 \mathrm{~h}$ intervals and perfused $24 \mathrm{~h}$ after the last injection. Survival and differentiation of newborn cells were tracked by the application of BrdU ( $50 \mathrm{mg} / \mathrm{kg}$ as above) twice per day ( $8 \mathrm{~h}$ intervals) for 3 consecutive days and perfusion 2 weeks after the first injection. Transcardial in-situ perfusions were performed in deeply anesthetized mice (40\% Esketaminhydrochlorid (Ketanest ${ }^{\circ}$, Pfizer, New York, USA), 20\% xylazine (Rompun ${ }^{\circ}$, Bayer, Leverkusen, Germany) in $0.9 \% \mathrm{NaCl}$ ) using $4 \%$ paraformaldehyde solution (PFA, Sigma-Aldrich, St. Louis, USA) in $0.1 \mathrm{M}$ phosphatebuffered $\mathrm{NaCl}$. Perfused brains were dissected out, kept overnight in $10 \mathrm{ml}$ of $4 \%$ PFA and 2 days in $30 \%$ sucrose solution at $4{ }^{\circ} \mathrm{C}$ after which whole brains were frozen in O.C.T. (Tissue-Tek Fisher Scientific, Hampton, USA) and stored at $-80^{\circ} \mathrm{C}$ until further processing.

\section{Immuno-fluorescence-histochemistry}

Coronal brain sections $(30 \mu \mathrm{m})$ containing the hippocampus were cut on a cryostat (CM1950, Leica, Wetzlar, Germany) using the Mouse Brain Atlas as a reference [27]. Free-floating brain sections were kept in a cryoprotectant solution (30\% glycerol, 30\% ethylene glycol and 40\% phosphate-buffered NaCl; pH 7.4). Every 10th section of the entire rostro-caudal span of the hippocampus was used for the quantification of newborn cells, their differentiation and survival using a published immunofluorescencehistochemistry procedure [24, 28-31]. For single staining (proliferation paradigm) only mouse anti-BrdU antibody (Bio-Rad AbD Serotec, United Kingdom; 1:300) was used. Double-staining protocols employed rat anti-BrdU antibody (Bio-Rad AbD Serotec, United Kingdom; 1:300) combined with goat anti-DCX (Santa Cruz, USA, 1:250), mouse antiNeuN (Millipore Merck, Germany, 1:1000) or rabbit antiGFAP (Sigma-Aldrich, St. Louis, USA, 1:500). Secondary antibodies were 488 donkey anti-goat (Thermofisher Scientific, Waltham, USA, 1:200), 488 goat anti-mouse (Thermofisher Scientific, Waltham, USA, 1:500), 488 goat anti-rabbit (Thermofisher Scientific, Waltham, USA, 1:500) and 594 chicken anti-rat (Thermofisher Scientific, Waltham, USA, 1:500).

Fluorescence microscopy pictures were acquired on a Carl-Zeiss Axiovert-Apotome System (Oberkochen, Germany) using the Axiovision software v4.8. For the evaluation of proliferation and survival the total number of BrdU+ cells in each hippocampal section (both hemispheres) was determined and a sum for each animal was 
calculated. Double-stainings in the survival paradigm were evaluated for the determination of cell differentiation based upon the number of cells co-expressing $\mathrm{BrdU}$ and either of the marker proteins (NeuN for mature neurons, GFAP for astrocytes). DCX stainings were used for the morphological characterization of newborn cells according to a previous study [31].

\section{Electrophysiological recordings}

Long-term potentiation (LTP) and basal synaptic transmission (Input/Output (I/O) curve and paired-pulse inhibition (PPI)) were evaluated in acutely dissected hippocampal slices. The procedures for the hippocampal slice preparation and electrophysiological recordings followed previously published protocols with some minor modifications [32]. Briefly, mice were killed and hippocampi were dissected under the microscope. Hippocampi were cut into $400 \mu \mathrm{m}$ thick slices using the Mcllwain Tissue Chopper (Mickle Laboratory Engineering, Guildford, Surrey, UK) and kept in artificial-cerebrospinal fluid (aCSF, in mM: $125 \mathrm{NaCl}, 2.5 \mathrm{KCl}, 25 \mathrm{NaHCO}, 2 \mathrm{CaCl} 2$, $1 \mathrm{MgCl} 2,25 \mathrm{D}$-glucose, $1.25 \mathrm{NaH} 2 \mathrm{PO} 4$ with $\mathrm{pH} 7.4)$, that was continuously bubbled with a 95/5\% mixture of $\mathrm{O}_{2} /$ $\mathrm{CO}_{2}$ to maintain physiological conditions. Slices were recovered for at least $1 \mathrm{~h}$ at $32{ }^{\circ} \mathrm{C}$ before recordings. Same physiological conditions were provided in the recording chamber with exception that picrotoxin was added at concentration of $30 \mu \mathrm{M}$ (Sigma-Aldrich, St. Louis, USA). Field excitatory postsynaptic potentials (fEPSPs) were recorded in the dentate gyrus (DG) with borosilicate glass pipette $(2-5 \mathrm{M} \Omega)$, pre-filled with aCSF solution, after stimulation of the medial perforant pathway (MPP). The stimulating bipolar electrode was made of teflon-coated tungsten wire that had a $50 \mu \mathrm{m}$ diameter and was located $\sim 400 \mu \mathrm{m}$ away from the recording electrode. The proper positioning of the electrodes was confirmed by applying a PPI protocol with $50 \mathrm{~ms}$ interstimulus interval at $30 \%$ of maximal voltage value that was determined by I/O curve. The PPI protocol consisted of a series of paired pulse stimulations with varying interstimulus intervals and used the ratio between second and first response as measurement. I/O curves were obtained by recording responses from repeated stimulation with pulses of voltage $1-9 \mathrm{~V}$ with interstimulus interval of $1 \mathrm{~V}$. The decaying slopes of field potentials were normalized to the maximum value of the slope. LTP was induced by high frequency stimulation, consisting of 4 trains of 100 pulses at $100 \mathrm{~Hz}$ with $15 \mathrm{~s}$ inter-train intervals. Synaptic potentiation was determined by analyzing the changes in the decaying phase of the fEPSP slopes normalized to the baseline values. Data were recorded by an AxoClamp-2B amplifier (bridge mode) and a Digidata-1440 interface (Axon Instruments, Foster City, USA). pClamp-10 (Molecular Devices, Biberach, Germany) was used for offline analysis.

\section{RNA sequencing}

After cervical dislocation, hippocampi were rapidly dissected on ice and total RNA was extracted using the miRNeasy mini kit (Qiagen, Hilden, Germany) according to the manufacturer's instructions including the optional DNase digest step (Qiagen, Hilden, Germany). RNA-Seq libraries were prepared with the TruSeq Stranded mRNA LT sample preparation kit (Illumina, San Diego, CA, USA) using Sciclone and Zephyr liquid handling workstations (PerkinElmer, Waltham, MA, USA) for pre- and post-PCR steps, respectively. For sequencing, samples were diluted and pooled into NGS libraries in equimolar amounts. Expression profiling libraries were sequenced on a HiSeq 3000 instrument (Illumina, San Diego, CA, USA) in 50-base-pair, single-end mode. Transcriptome analyses were performed with the Tuxedo suite. For each sample, reads passing vendor quality filtering were aligned to the $\mathrm{mm} 10$ reference genome assembly provided by the UCSC Genome Browser based on Genome Reference Consortium GRCm38 with TopHat2 (v2.1.1, [33]). Cufflinks (v2.1.1, [34]) allowed for transcriptome assembly on the basis of the reference transcriptome and spliced read alignments, as well as raw transcript quantification. Cuffdiff was used for differential expression calling. Transcripts with a false discovery rate $\mathrm{FDR} \leq 0.05$ and a $\log _{2}$-fold change $\geq+1.5$ or $\leq-1.5$ for up- and downregulated genes, respectively, were considered as significantly differentially expressed transcripts (DETs).

Gene ontology enrichment analysis was performed for biological pathway, cellular compartment and molecular function sub-ontologies using the freely available software tools DAVID [35, 36] and Enrichr [37, 38].

\section{Neuropathohistology}

Gross neuropathohistological assessment was carried out on coronal brain sections stained with hematoxylin/ eosin (H\&E) followed by immunohistochemical assays suitable for the examination of the integrity of neuronal cell bodies, dendrites and axons (anti-phosphorylated neurofilament-H, SMI31 and anti-non-phosphorylated neurofilament-H, SMI32). Briefly, after transcardial perfusion, frozen brains were cut on a brain slicer (Zivic Instruments, Pittsburgh, USA) into $2 \mathrm{~mm}$ sections and embedded into paraffin. The tissue blocks were cut into $4 \mu \mathrm{m}$ slices and automated immunohistochemistry was performed on Autostainer 48 Link instruments (Dako, Glostrup, Denmark) using EnVision ${ }^{\mathrm{Tm}}$ FLEX+ detection system (Dako, Glostrup, Denmark) according to the manufacturer's recommendations. All primary antibodies were incubated for $30 \mathrm{~min}$ at room temperature using a dilution of 1:50000 for anti-SMI-31 (Biolegend, San Diego, USA) and 1:200 for anti-SMI-32 (Biolegend, San Diego, USA). All sections were counterstained with 3,3'- 
diaminobenzidine (DAB, Merck, Darmstadt, Germany) for specific labeling and Mayer's hemalum solution (Merck, Darmstadt, Germany) for nuclear labeling. Images were scanned on a Pannoramic midi Scanner (3DHISTECH Ltd., Budapest, Hungary) and qualitatively analyzed using Pannoramic Viewer (3DHISTECH Ltd., Budapest, Hungary).

For the validation of myelin transcripts Mouse on Mouse Immunodection kit (MOM, Vector laboratories, Burlingame, USA) was used, employing the following primary antibodies: anti-MOG (myelin oligodendrocyte glycoprotein, Sigma-Aldrich, St. Louis, USA, 1:1000), anti-PLP1 (myelin proteolipid protein, BioRad, Hercules, USA, 1:500) and anti-CNP $\left(2^{\prime}, 3^{\prime}\right.$-Cyclic-nucleotide $3^{\prime}$ phosphodiesterase, Biolegend, San Diego, USA, 1:1000). The intensity of the DAB signal in MOG and PLP1 stainings was automatically determined in a selected region of interest within the hippocampus using Qupath [39]. For the number of oligodendrocytes, CNP+ cells were manually counted in a selected region of interest within the hippocampal dentate gyrus.

\section{Mitochondrial respiratory chain enzymatic activities}

Enzymatic activities of mitochondrial respiratory complexes I - IV and citrate synthase (as reference enzyme) were tested using a standard published method [40]. Snap-frozen hippocampal tissue was homogenized with a glass-glass tissue grinder and centrifuged at $600 \mathrm{G}$ for $10 \mathrm{~min}$ at $4{ }^{\circ} \mathrm{C}$ to isolate mitochondria. Supernatant was aliquoted to avoid multiple freeze-thaw cycle. Total protein concentration was measured using Bradford assay (BioRad, Hercules, USA). Enzymatic activity measurements were conducted at a constant temperature of $37^{\circ} \mathrm{C}$ using Shimadzu Spectrophotometry (UV-1800 Shimadzu, Kyoto, Japan).

\section{Mitochondrial membrane potential}

Primary hippocampal neurons were prepared from postnatal day 1 WT and KI pups according to previously described protocol with some minor modifications [41]. Briefly, mouse hippocampi were isolated and cells were cultured at a density of 50,000 cells $/ \mathrm{cm}^{2}$ on poly-D-lysine coated glass-bottom dishes $(35 \mathrm{~mm})$ using Neurobasal A medium supplemented with $2 \%$ B27, $0.5 \mathrm{mM}$ glutamax and penicillin/streptomycin $(10,000 \mathrm{U} / \mathrm{ml})$. Cells were maintained for 2 weeks at $37{ }^{\circ} \mathrm{C}$ with $5 \% \mathrm{CO}_{2}$.

The mitochondrial membrane potential $\left(\Psi_{\mathrm{m}}\right)$ was measured using a fluorescence-based method as previously published with some adaptions in concentration and laser intensities [42]. Briefly, cells were equilibrated for $1 \mathrm{~h}$ at $37^{\circ} \mathrm{C}$ in external buffer containing $1 \mathrm{nM}$ TMRM to avoid fluorescence quenching. The same concentration of TMRM was present in all perfusing solutions. The external buffer contained: $140 \mathrm{NaCl}, 3 \mathrm{KCl}, 2 \mathrm{CaCl} 2,2 \mathrm{MgCl} 2$,
10 HEPES, 20 glucose (in mM, pH 7.4). All chemicals were purchased from Thermofisher Scientific, Waltham, USA. Pharmacological intervention with ATP synthase inhibitor (Oligomycin), complex III inhibitor (Antimycin A) and protonophore (FCCP) were made (all substances ordered from Sigma-Aldrich, St. Louis, USA).

After loading, cells were imaged using a confocal microscope (Nikon A1r, Minato, Japan) by excitation/emission wavelengths of $562 \mathrm{~nm} / 595 \mathrm{~nm}$. Experiments were performed at room temperature and cells were continuously locally superfused using an 8-reservoir drug application system (Octaflow II). Image analysis was conducted by specifying mitochondria located in neuronal bodies as region of interest and the mean fluorescence intensity profile over time was calculated. All images were quantified using open access ImageJ (Fiji) [43].

\section{Statistical analyses}

Sample sizes were determined according to previous studies $[24,44,45]$. All data were tested for normality using the Kolmogorov-Smirnov test prior to further statistical evaluation. Cosinor analysis was performed to determine circadian oscillations. Data were analyzed using CircWave statistic software (V1.4) according to the eq. $\mathrm{Y}=\mathrm{c}+\mathrm{aSIN}(\mathrm{i} 2 \pi t / 24)+\mathrm{bCOS}(\mathrm{i} 2 \pi t / 24)$, where $\mathrm{Y}$ is delta cycle threshold, $t$ is circadian time, and a, b, and c were predicted. Further nonlinear regression analysis was used to determine peak time, trough and amplitude of the curve, using the center of gravity parameter. Behavioral experiments were analyzed using 2-way ANOVA, repeated measure ANOVA and 3-way ANOVA. Neurogenesis and mitochondrial results were evaluated by Student's t-test or corrected with Welch's ttest, where appropriate. Electrophysiological data were analyzed using repeated measure ANOVA. Statistical outliers (values outside of the interval: mean \pm 2 standard deviations) were excluded from further analyses. The threshold for significance was set at $" p<0.05$ in all instances. Statistical analyses were performed using SPSS software for Windows, Version 24. (IBM Corporation, Chicago, USA) and Graphpad Prism, Version 7 (San Diego, USA).

\section{Results}

\section{Characterization of emotional behavior in KI mice}

We initially analyzed the behavioral phenotype of HD-AIP mice comparing adult male and female KI animals to wildtype littermate controls, focusing on depression- and anxiety-like displays, in light of the symptoms reported in AIP patients. In consideration of the previously described motoric deficiencies in the KI mice, particularly during the neonatal and early postnatal period [13], we first examined overall locomotor activity in adult animals to exclude a possible bias in the subsequent behavioral paradigms. Long-term ( $24 \mathrm{~h}$ ) assessment of home cage activity revealed no genotype effect on total mobility in male 


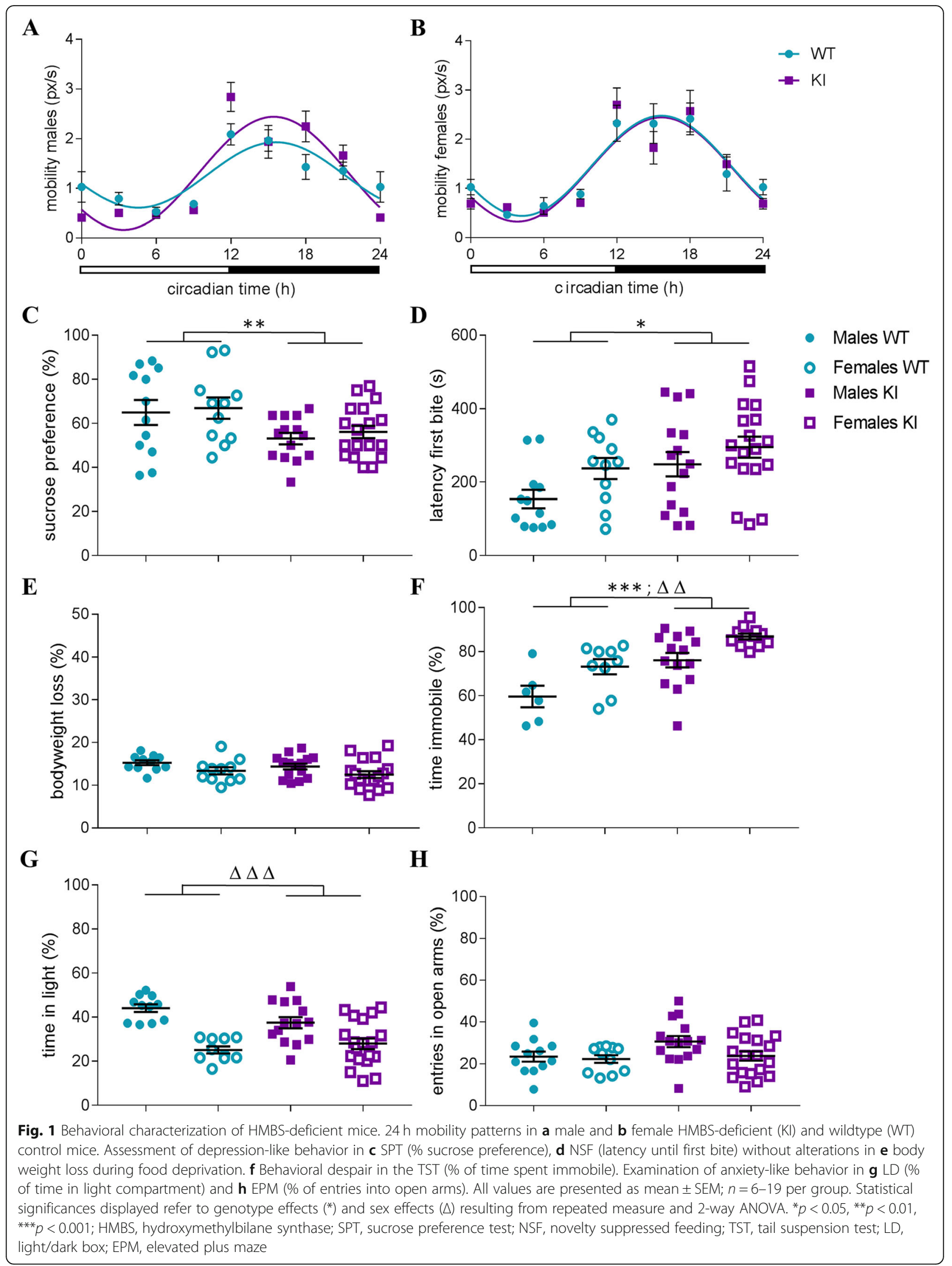


and female KI mice (Fig. 1a-b). Subsequent cosinor analysis documented statistically significant $24 \mathrm{~h}$ rhythmic patterns of mobility for all groups $(p<0.0001)$. These data excluded locomotor impairment as a liability for the behavioral assessment of adult KI mice and allowed us to proceed to mood-specific tests.

Male and female KI mice showed reduced sucrose preference compared to their respective controls in the SPT, indicating augmented depression-related anhedonic behavior (Fig. 1c; significant main effect of genotype $(F(1,51)=$ 8.37, $p<0.01)$. In line with this observation, enhanced depression-related anxiety in the KI mice was revealed in the NSF test as evidenced by a longer latency to the first bite (Fig. 1d; significant main effect of genotype $(F(1,52)=6.24$, $p<0.05$ ). Importantly, no significant differences between genotypes were observed in the amount of bodyweight loss after a $24 \mathrm{~h}$ food restriction (Fig. 1e). Furthermore, there was no correlation between home cage food consumption (5 min after the NSF) and the latency to the first bite in the NSF ( $R^{2}=0.048$ for males, $R^{2}=0.076$ for females), collectively excluding an unspecific bias in the NSF due to metabolic alterations and/or motivation to eat. In agreement with the previous results, immobility in the TST, commonly used as an indicator for behavioral despair in the context of depression-like behavior, was elevated in male and female KI mice (Fig. 1f; significant main effect of genotype ( $\mathrm{F}(1$, $37)=20.62, p<0.0001)$; significant main effect of sex $(\mathrm{F}(1$, $37)=13.31, p<0.001)$ ). No alterations in anxiety-like behavior were detected in the KI mice in comparison to WT controls in the LD (time in the light zone) or the EPM (open arm entries). A significant main effect of sex $(F(1,50)=$ $35.05, p<0.0001)$ for the time spent in light compartment was observed in the LD (Fig. 1g-h).

In the TST, depression-like behavior was reduced by acute treatment with the selective serotonin reuptake inhibitor (SSRI) Escitalopram (10 mg/mg i.p.) in mice of both genotypes (reduction in \% immobility (mean \pm SEM: KI males $78.44 \pm 2.56 \%$ to $43.60 \pm 6.19 \%$; KI females $86.84 \pm 1.14 \%$; WT males to $53.00 \pm 8.61 \%$; WT males $59.63 \pm 4.00 \%$ to $23.13 \pm 3.72 \%$; WT females: $73.12 \pm 4.66 \%$ to $44.13 \pm 7.38 \%$ ).

Since no sex $\times$ genotype interactions were observed for any of the behavioral tests, samples were collapsed across sexes for all following experiments. The number of animals of each sex used for the individual analyses as well as total sample sizes are summarized in Additional file 1.

\section{Myelination related transcripts are differentially expressed in the hippocampus of KI mice}

Based on the depression-like behavior detected in the KI mice, our subsequent analyses focused on the hippocampus, a limbic brain structure central to the pathophysiology of depression [46-48].

We performed high-throughput RNA sequencing (RNA$\mathrm{Seq}$ ) in order to explore the molecular signature accompanying the behavioral deficits in the KI mice in an unbiased manner. Three hundred twenty transcripts were found to be significantly and more than 1.5 -fold differentially expressed between genotypes. A list of all differentially expressed genes (DEGs) is provided in Additional file 2. Raw data are available at www.ncbi.nlm.nih.gov/geo/query/ acc.cgi?acc=GSE146212.

Bioinformatic analyses focusing on biological pathways, cellular compartments and molecular function subontologies revealed a notable enrichment of DEGs for CNS myelination and oligodendrocyte development (Fig. 2a). To gain further insight into the possible pathophysiological implications of the molecular alterations in the KI hippocampus, differentially expressed genes in KI mice were contrasted against available gene sets from DisGeNet [49] with relevance for depression and myelin deficits. Three genes were detected in the three-way comparison: Myelin

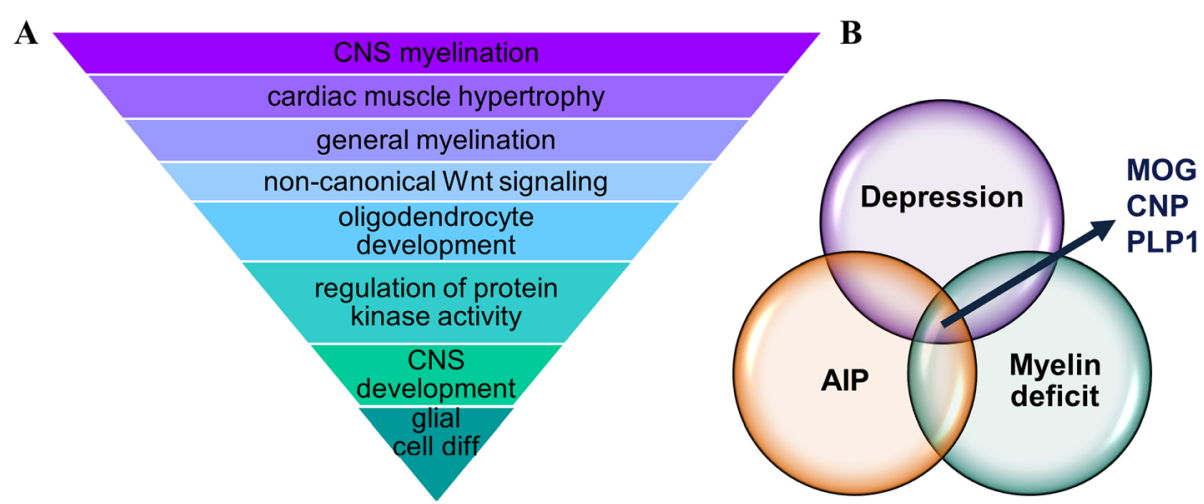

Fig. 2 High-throughput RNA sequencing of hippocampal tissue of HMBS-deficient mice. a Results of enrichment analysis revealing a gene expression signature with relevance for myelination in KI mice. $\mathbf{b}$ Venn-diagram highlighting 3 genes found to be differentially expressed in $\mathrm{Kl}$ as compared to WT mice which are also involved in depression and myelin deficits ( $n=6$ per genotype). AIP, acute intermittent porphyria; MOG, myelin oligodendrocyte glycoprotein; CNP, 2',3'-Cyclic-nucleotide 3'-phosphodiesterase; PLP1, myelin proteolipid protein 1 (CNS) 
Oligodendrocytes Glycoprotein (MOG), 2',3'-Cyclic-nucleotide 3 '-phosphodiesterase (CNP) and Proteolipid Protein 1 (PLP1) (Fig. 2b).

\section{$\mathrm{KI}$ mice present with defective myelination and a reduction in oligodendrocytes number}

In order to validate and extend the RNA-Seq results, hippocampal protein levels of MOG, PLP-1 and CNP were examined immunohistochemically. In accordance with the results at the mRNA level, the intensity of the 3,3'-Diaminobenzidine (DAB) staining revealed a significant decrease in MOG (Fig. 3a-b; t $(12.52)=5.18, p<0.001)$ and PLP1 $(\mathrm{t}(16)=2.35, p<0.05)$ protein expression in KI mice (Fig. 3c-d). A significant difference between genotypes was also observed for the number of $\mathrm{CNP}+$ cells (Fig. 3e-f; $\mathrm{t}(17)=2.49, p<0.05)$, indicating fewer oligodendrocytes in $\mathrm{KI}$ than in control littermates.

\section{Altered mitochondrial activity in KI mice}

Subsequent efforts explored mitochondrial dysfunction as a possible pathophysiologic link between HMBS-deficiency and its impact on affective behavior and myelination. ALA has been previously shown to detrimentally impact myelination in the peripheral nervous system, and this effect was proposed to result from mitochondrial respiratory chain defects and ensuing oxidative damage [50,51].

We therefore determined the activity of the four mitochondrial respiratory chain complexes (CI-CIV) individually in mitochondria isolated from KI and WT hippocampal tissue using citrate synthase as a quantitative marker enzyme. No significant difference between genotypes for CI (NADH: ubiquinone oxidoreductase) and CII (succinate dehydrogenase) activities were found (Fig. 4a and b). However, for the heme containing complexes CIII (cytochrome c reductase) and CIV (cytochrome c oxidase) (Fig. 4c and d), a significant decrease of enzymatic activity $(\mathrm{t}(17)=3.257, p<0.01$ and $\mathrm{t}(17)=3.867, p<0.01)$ in KI mice compared to WT littermates was revealed.

Reduced activity of these respiratory complexes may result in lower efficiency of mitochondrial respiration. Therefore, we measured mitochondrial membrane potential $\left(\Psi_{\mathrm{m}}\right)$, which is directly controlled by the activity of respiratory chain complexes and the activity of mitochondrial ATP synthase, in-vitro using primary hippocampal neurons from KI and WT mice. Mitochondrial function is coupled to the state of polarization [52] and indeed our data demonstrate that mitochondria of KI neurons were less polarized (lower TMRM intensity) compared to those of WT neurons (Fig. 4e; $\mathrm{t}(19)=4.29, p<0.001)$. In the next step we examined $\psi \mathrm{m}$ maintenance. Mitochondria with normal respiratory activity maintain the $\psi \mathrm{m}$ by the proton pumping activity of the respiratory chain while ATP synthase couples a depolarizing proton influx to the ATP synthesis. However, in the case of reduced respiratory function, ATP synthase may reverse its mode of operation and pump protons out of the mitochondrial matrix, thus maintaining $\psi \mathrm{m}$ at the expense of ATP. Incubation with the ATP-synthase inhibitor Oligomycin $(1 \mu \mathrm{M})$ did not change the TMRM fluorescence in WT mitochondria, while the subsequent application of the complex III inhibitor Antimycin A $(2 \mu \mathrm{M})$, led to the expected gradual decrease of TMRM fluorescence, indicating that in the mitochondria of WT neurons, $\Psi_{\mathrm{m}}$ is maintained by the activity of respiratory chain (Fig. 4f). In contrast, addition of Oligomycin resulted in a decrease of TMRM fluorescence in the mitochondria of KI neurons, while the subsequent addition of Antimycin A exerted only a modulatory effect on the ongoing depolarization. Protonophore FCCP $(2 \mu \mathrm{M})$ was added to achieve a maximal depolarization at the end of the experiments in order to demonstrate that the measured fluorescence originated from mitochondria. See Additional file 3 for the confirmation of the localization of TMRM in mitochondria in original micrographs.

\section{Adult hippocampal cell proliferation and differentiation is impaired in KI mice}

To further explore the consequences of disrupted mitochondrial energy metabolism on hippocampal function, we focused on its neurogenic and neuroplastic potentials, both of which have been also repeatedly linked to depression-like behavior.

BrdU administration in the proliferation and survival paradigms was used to investigate dentate gyrus neurogenesis (Fig. 5a). Quantification of BrdU+ cells, revealed a highly significant reduction in hippocampal progenitor cell proliferation in the KI mice compared to WT littermates (Fig. 5b-c; $t(20)=6.98, p<0.001$ ). However, the rate of survival of newborn cells was comparable among genotypes (Fig. 5d). Subsequent co-staining with astrocytic (GFAP) and neuronal (NeuN) markers for the evaluation of cell fate demonstrated no difference in the relative amount of cells differentiated into astrocytes (Fig. 5e), but a significant decrease $(\mathrm{t}(17.25)=3.80, p<0.01)$ in mature neurons among all BrdU+ cells in the KI mice was observed (Fig. 5f; NeuN+ cells co-labeled with BrdU).

To gain deeper insight into the process of neuronal differentiation in adult-born cells, morphologically distinct stages of neural maturation [31] were examined using doublecortin (DCX) stainings in BrdU+ cells (Fig. 5g). Here, a selective deficit in the differentiation into fully mature, postmitotic neurons was detected in the KI mice, as indicated by a higher percentage of BrdU+ cells in the early proliferative $(\mathrm{t}(14.23)=2.64, p<0.05)$ and intermediate phases $(\mathrm{t}(25)=2.25, p<0.05)$ and a lesser percentage in the postmitotic stage $(\mathrm{t}(12.42)=2.70, p<0.05)$ than in WT littermates (Fig. 5h).

To further explore the consequences of disrupted mitochondrial energy metabolism on hippocampal function, we focused on its neurogenic and neuroplastic potentials, both 


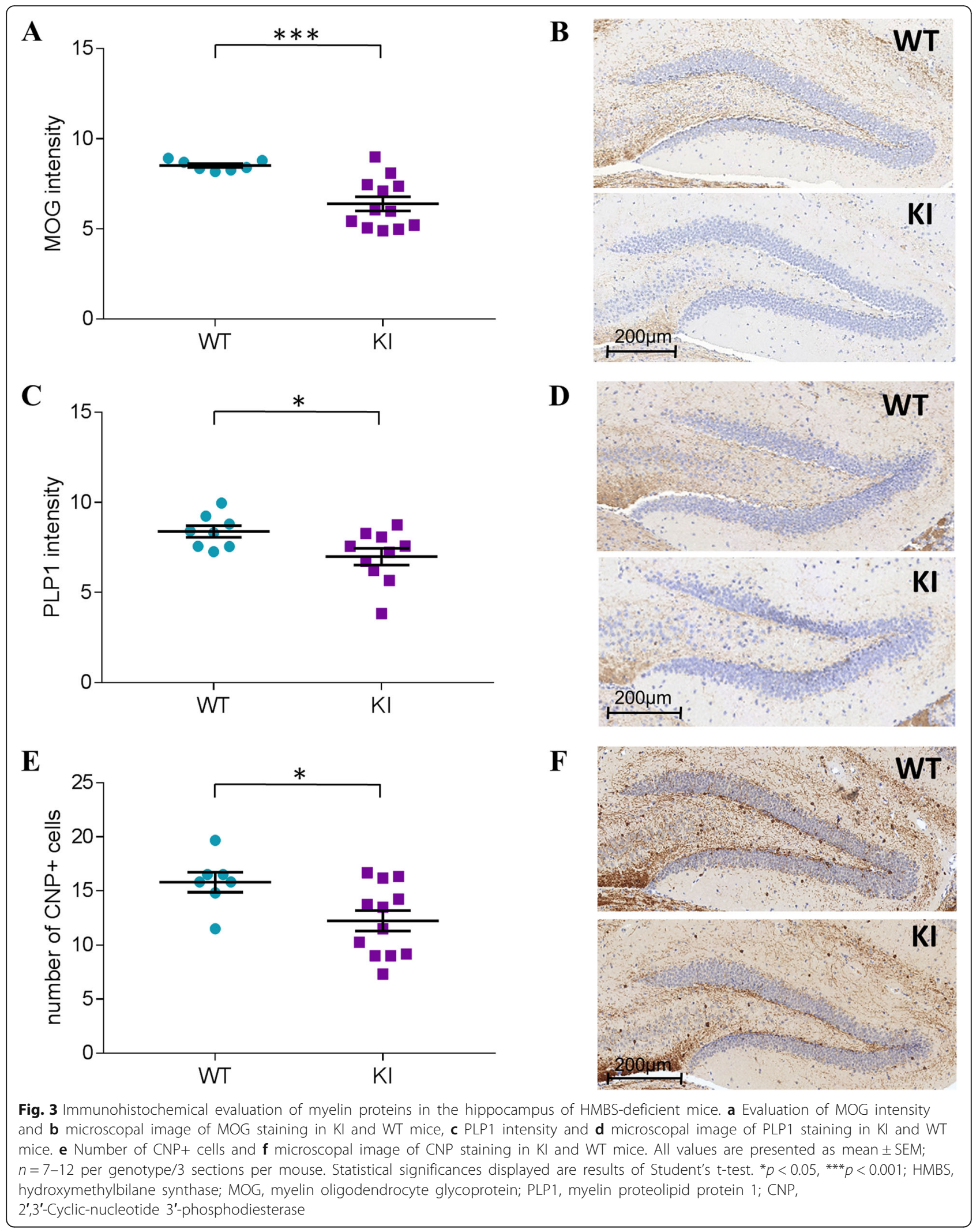




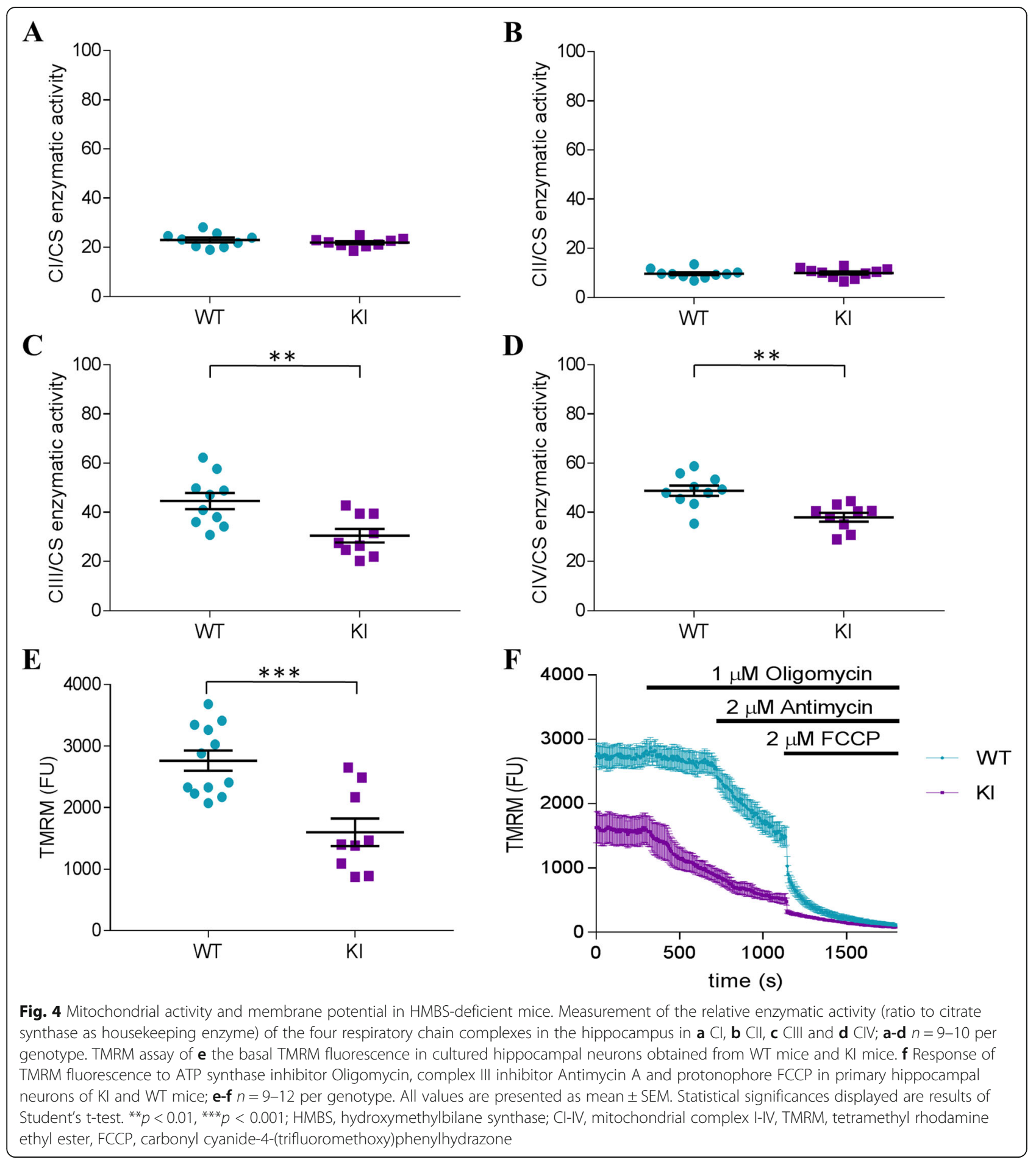

of which have been also repeatedly linked to depressionlike behavior.

\section{Aberrant synaptic plasticity parallels behavioral and neurogenic deficits in $\mathrm{KI}$ mice}

We next interrogated hippocampal synaptic plasticity by assessing long-term potentiation as a form of activity-dependent enhancement of synaptic strength. We found that stimulation of the medial perforant path (Fig. 6a) resulted in a significantly blunted response (i.e., potentiation) in dentate gyrus to high frequency stimulation (HFS) in the KI mice compared to WT littermate controls (Fig. $6 \mathrm{~b}$ and c; $\mathrm{F}(1,40)=4.46$, $p<0.05)$. 
A

Proliferation paradigm

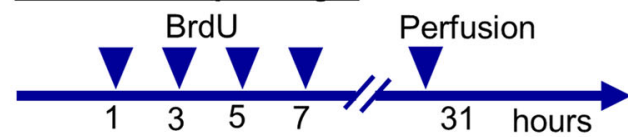

Survival paradigm

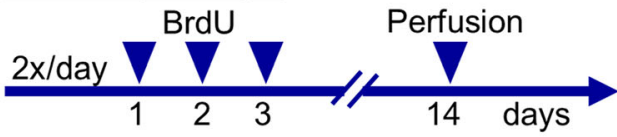

C
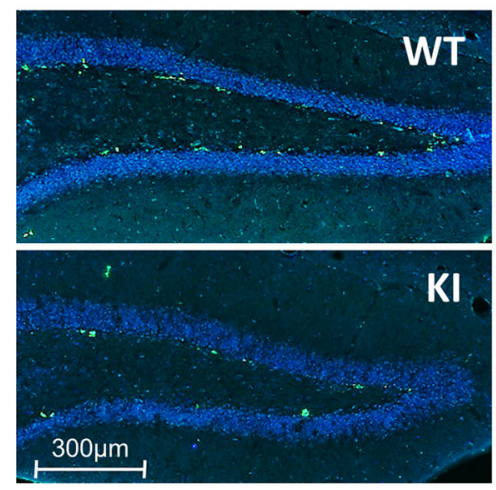

$\mathbf{E}$

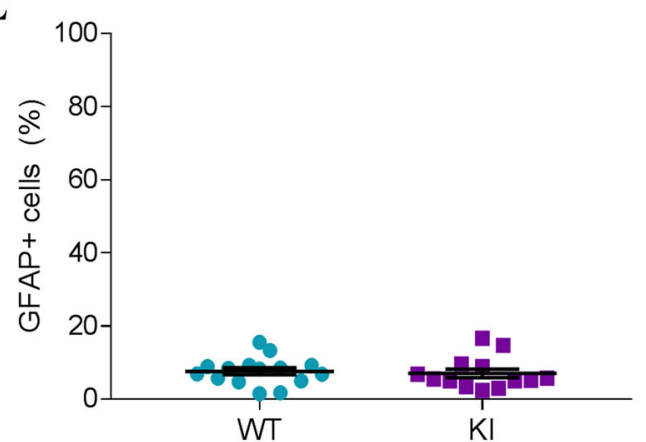

G

\begin{tabular}{|c|c|c|}
\hline 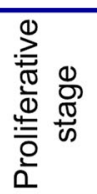 & $\begin{array}{l}\text { No or short } \\
\text { processes }\end{array}$ & $\stackrel{30 \mu \mathrm{m}}{2}$ \\
\hline 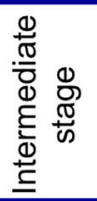 & $\begin{array}{l}\text { Medium } \\
\text { processes } \\
\text { reaching } \\
\text { molecular layer }\end{array}$ & \\
\hline 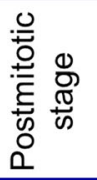 & $\begin{array}{l}\text { Dendritic branching } \\
\text { in molecular and } \\
\text { granule cell layer }\end{array}$ & \\
\hline
\end{tabular}

B

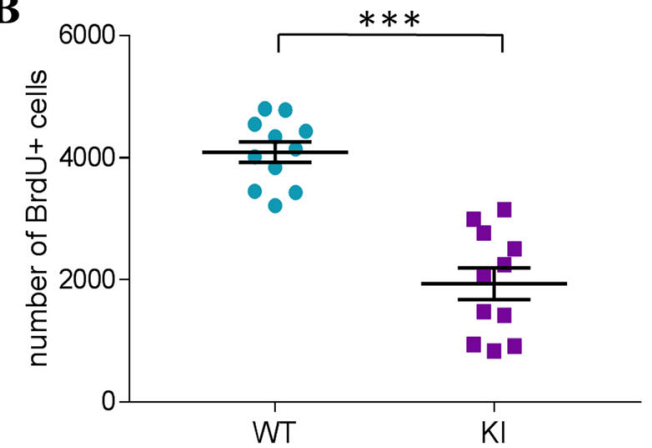

D

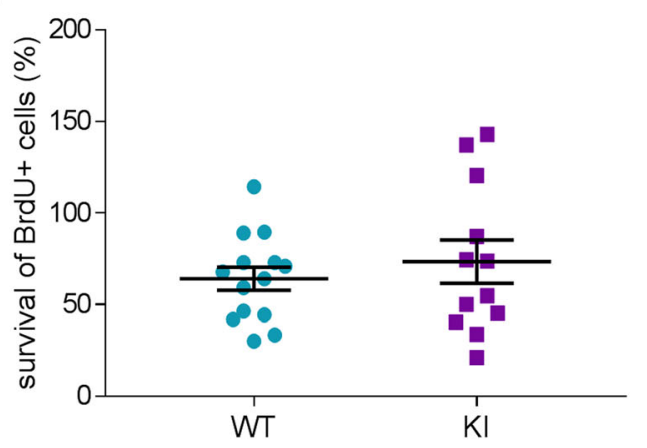

$\mathbf{F}$

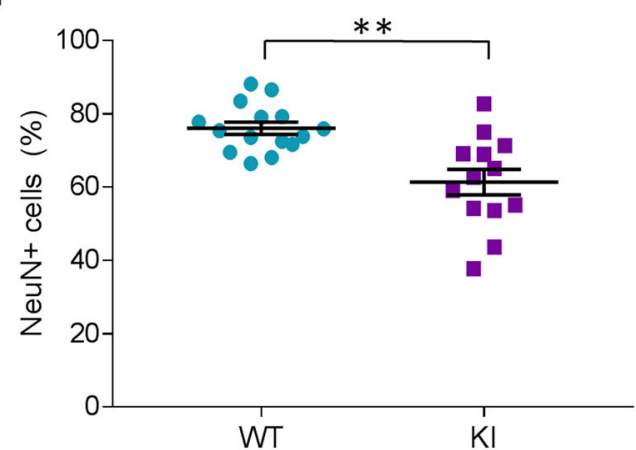

H

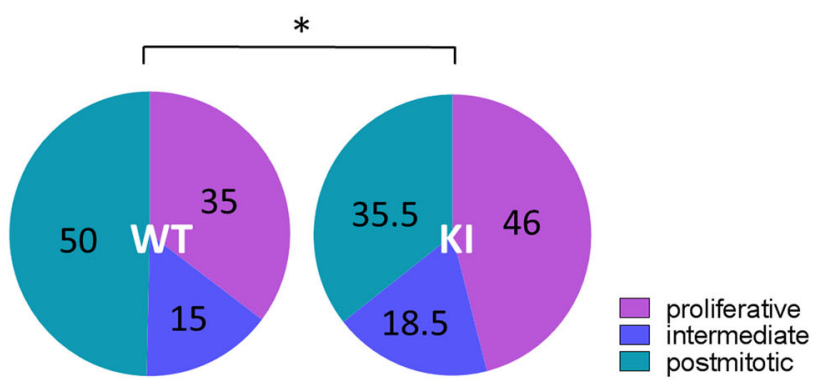

Fig. 5 (See legend on next page.) 
(See figure on previous page.)

Fig. 5 Analysis of adult hippocampal neurogenesis in HMBS-deficient mice. a BrdU injection schedules for the proliferation and survival paradigms. b Quantification of hippocampal progenitor cell proliferation (BrdU+ cell count) and $\mathbf{c}$ respective fluorescence microscope images in HMBS-deficient (KI) and wildtype (WT) mice (BrdU+ cells in green). d Survival of newborn (BrdU+) cells over a two-week period. e Relative differentiation rates of newborn (BrdU+ cells) cells into astrocytes (GFAP+ cells) and $\mathbf{f}$ mature neurons (NeuN+ cells). $\mathbf{g}$ Developmental stages of doublecortin stained DCX+ (green) BrdU+ (red) labeled cells according to defined morphological criteria (proliferative, intermediate and postmitotic). $\mathbf{h}$ Quantification (\%) of BrdU+ cells in each developmental stage in $\mathrm{Kl}$ and WT mice. All values are presented as mean $\pm \mathrm{SEM} ; n=11-$ 13 per genotype/3 sections per mouse. Statistical significances displayed are results of Student's t-tests. ${ }^{* *} p<0.001,{ }^{* *} p<0.01,{ }^{*} p<0.05 ; H M B S$, hydroxymethylbilane synthase; BrdU, 5-bromo-2'-deoxyuridine; GFAP, glial fibrillary acidic protein; NeuN, neuronal nuclei; DCX, doublecortin

Basal synaptic function was intact in the KI hippocampus, as reflected in comparable slopes of input-output recordings between genotypes (Fig. 6d). We further found the alterations in synaptic plasticity in the KI mice to be primarily relevant for long-term synaptic plasticity, as no genotype effect was notable in paired-pulse inhibition recordings, representative of short-term plasticity events (Fig. 6e).

Using immunohistochemical stainings for standard markers for neuronal integrity (H\&E, SMI-31 and SMI32), gross neuropathohistological defects were excluded as non-specific biases of the electrophysiological and behavioral results (Additional file 4).

\section{Discussion}

In a comprehensive characterization of the HD-AIP mouse model we revealed, for the first time, augmented depression-like behavior as a consequence of severe, constitutive HMBS deficiency. This phenotype was confirmed using three independent paradigms routinely employed for the evaluation of depression-like behavior in mice, excluding task-specific performance as artifacts and highlighting the validity of the findings. Importantly, augmented immobility in the TST was reduced by acute administration of Escitalopram, suggesting SSRI treatment as possible therapeutic strategy for AIP patients suffering from affective symptomatology. Along these lines it will be interesting to explore in future studies whether and how the behavioral consequences of severe HMBS deficiency interact with the effects of acute or chronic stress exposure and the related sensitivity to pharmacotherapy.

As previous study revealed motor deficits of the KI mice [13], a possible bias as a result of hypolocomotion was ruled out by $24 \mathrm{~h}$ continuous activity monitoring. Considering that psychiatric manifestations are often associated with dysregulation of circadian rhythms [53] it is noteworthy that the circadian rhythmicity of daily activity was preserved in KI mice.

Interestingly, despite the robust augmentation of depression-like behavior in the KI mice, no effect on anxiety-like behavior was observed. One can speculate that the brain regions more relevant to the neural circuitry of depression than of anxiety are specifically vulnerable to the pathophysiological sequelae of accumulation of ALA and
PBG. This consideration led us to focus our subsequent interrogation on the hippocampus, due to its relevance for the pathophysiology of depression [46-48]. However, we cannot exclude that other brain regions may also be affected/ involved. We employed RNA-Seq analysis as hypothesis-free approach to explore the molecular consequences of severe HMBS-deficiency in the KI hippocampus. Bioinformatic workup of differentially expressed transcripts revealed impaired myelination and oligodendrocyte development emerged as relevant biological theme. When we specifically analyzed differential gene expression in KI mice, genes that have been previously linked to myelination deficits and depression-like states, MOG, PLP1 (myelin markers) and CNP (oligodendrocytic marker) were implicated as molecular players. Subsequent immunohistochemical analyses corroborated these findings at the protein level, further supporting the role of defective myelination as a potential pathogenic mechanism in the behavioral, neurogenic and plasticity defects in KI mice. Indeed, evidence from previous animal studies, post-mortem human studies, as well as genetic and neuroimaging experiments indicate an association between major depressive disorder (MDD) and changes in brain myelin content [54]. Interestingly, patients with neurological disorders of the white matter, like multiple sclerosis (MS) often show psychiatric symptoms, including depression, and display comparable dysregulation in gene expression $[55,56]$. In line with our observations, myelin deficits have been revealed in the seminal characterization of the (HD-AIP) KI mice [13] and have also been reported in AIP patients [57]. However, the mechanism by which HMBS deficiency may alter CNS myelination has remained elusive to date.

Disturbed mitochondrial energy production is frequently observed in congenital diseases and often related to neurological and psychiatric symptoms [58, 59]. Taking into consideration that half of the heme biosynthetic pathway is processed in the mitochondria and that disturbed heme metabolism causes mitochondrial defects $[50,51]$, a possible direct link between AIP and mitochondrial dysfunction is plausible. Indeed, impairments of mitochondrial energy metabolism in the liver, muscle and brain have also been demonstrated in the T1/T2 mouse model following phenobarbital administration $[50,51]$. We tested the impact of HMBS deficiency on 


\section{A}

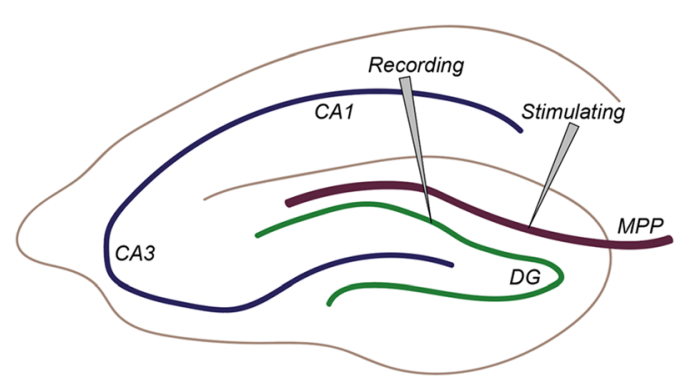

B

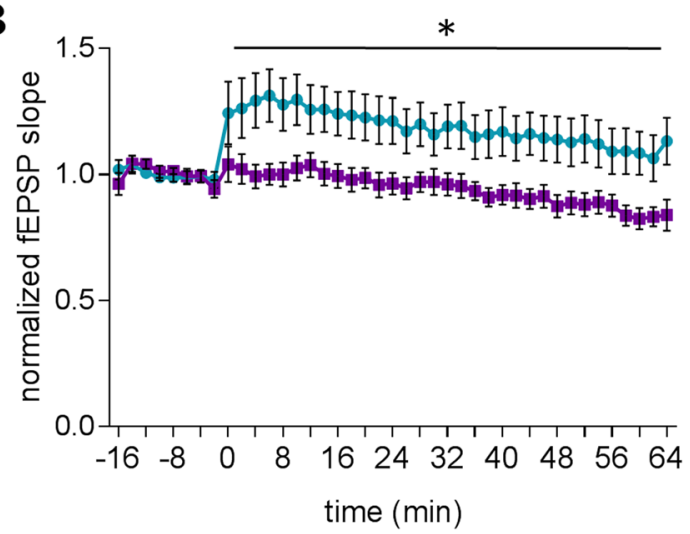

C
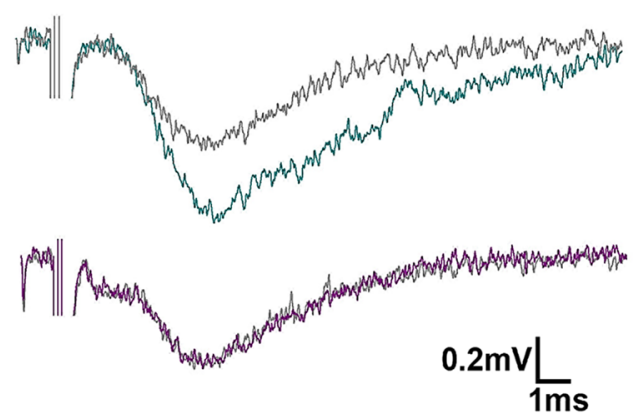

E

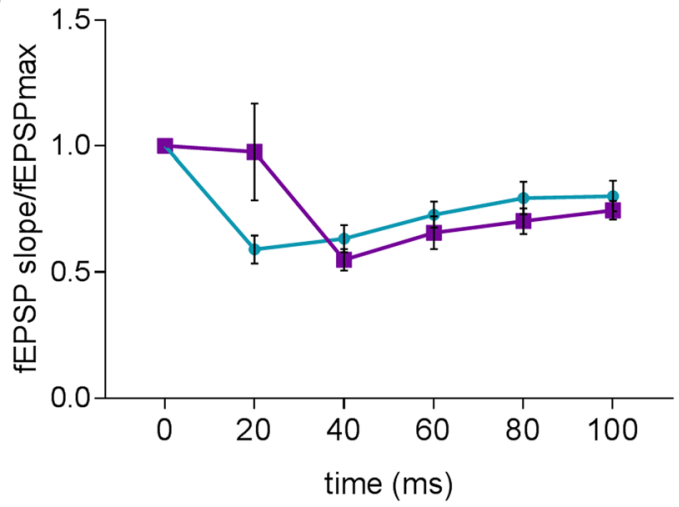

D

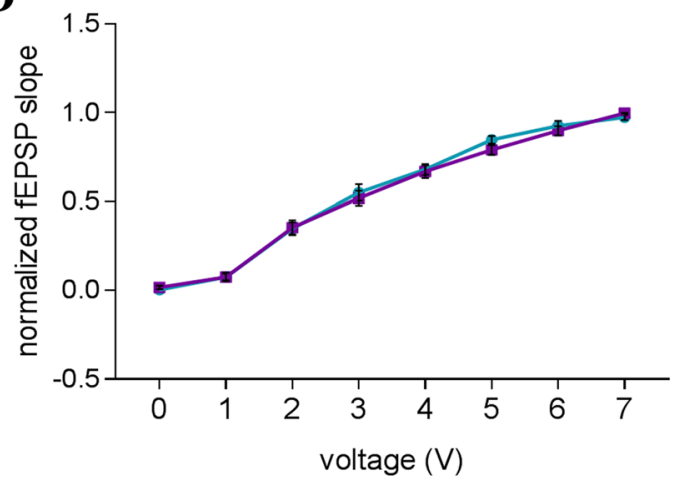

Fig. 6 Electrophysiological evaluation of hippocampal dentate gyrus synaptic plasticity in HMBS-deficient mice. a Schematic depiction of the placement of the electrodes for LTP recordings. $\mathbf{b}$ LTP of fEPSP after high frequency stimulation and $\mathbf{c}$ representative traces in HMBS-deficient (KI) and WT mice. $\mathbf{d}$ Input-output recordings and $\mathbf{e}$ paired-pulse inhibition of fEPSP to maximum fEPSP. All values are presented as mean \pm SEM; $n=$ 8-12 per genotype. Statistical significances displayed reflect results of repeated measure mixed model ANOVA. ${ }^{*} p<0.05$; HMBS,

hydroxymethylbilane synthase; LTP, long-term potentiation; fEPSP, field excitatory postsynaptic potential, MPP, medial perforant path; DG, dentate gyrus

mitochondrial function in the KI mouse model, selectively focusing on the hippocampus. While enzymes of the mitochondrial complexes $\mathrm{CI}$ and $\mathrm{CII}$ had comparable activity among genotypes, there was a significant decrease of enzymatic activity in complexes CIII and CIV in the KI mice compared to controls. Involvement of heme in the function of CII has been proposed, however its role is unclear to date. For CIII and CIV, the relevance of heme for electron transport and reduction of molecular oxygen, respectively, have been delineated in detail (for review see [60]). Hence, reduced heme availability in HMBS-deficient mice is likely to cause a selective impact on CIII and CIV activity.

The reduction in the enzymatic activity of the respiratory chain in hippocampal tissue was strengthened by the result of decreased mitochondrial membrane potential in primary 
hippocampal KI neurons. These results further indicate that the KI mitochondria were characterized by insufficient respiratory activity and maintained their membrane potential by reverse ATP synthase, resulting in ATP consumption rather than production. The reversal potential of ATP synthase activity is typically only a few $\mathrm{mV}$ below the resting mitochondrial membrane potential. Thus, even a mild decrease of mitochondrial membrane potential may be sufficient to reverse the action of ATP synthase [61].

To determine the consequences of disrupted mitochondrial energy metabolism on hippocampal function, we analyzed dentate gyrus neurogenesis and synaptic plasticity. Neurogenic aberrations have been reported in a variety of different animal models of depression [46-48, 62]. Proliferating and newly born cells are more susceptible to the lack of energy supply resulting from impaired mitochondrial function (for review see [63]). Along these lines we here found an impact of HMBS-deficiency specifically on dividing progenitor cells but not on postmitotic neurons or other cell types, as no gross histopathological alterations were observed in the KI hippocampus. Mechanisms of synaptic plasticity, including LTP, are also critically dependent on mitochondrial function (for review see [64]) and are deranged in several animal models of depression $[65,66]$. Indeed, we here observed impaired activity-dependent synaptic strengthening in the KI dentate gyrus, which may be caused by mitochondrial dysfunction.

Aberrant mitochondrial function is increasingly recognized as an important element in the pathophysiological mechanisms of depression (for review see [59]) and physiological mitochondrial activity is pivotal for oligodendrocyte function and myelin production [67, 68]. These considerations close the loop to the affective and myelination deficits in KI mice and collectively suggest compromised mitochondrial function as pathogenetic mechanism contributing to the behavioral and neuropathological manifestations in the HMBS-deficient mouse model of HD-AIP (Fig. 7).

Thus, while HD-AIP and AIP are distinct metabolic disorders, our KI mice and phenobarbital-induced T1/T2 mice both display significant mitochondrial energetic defects in

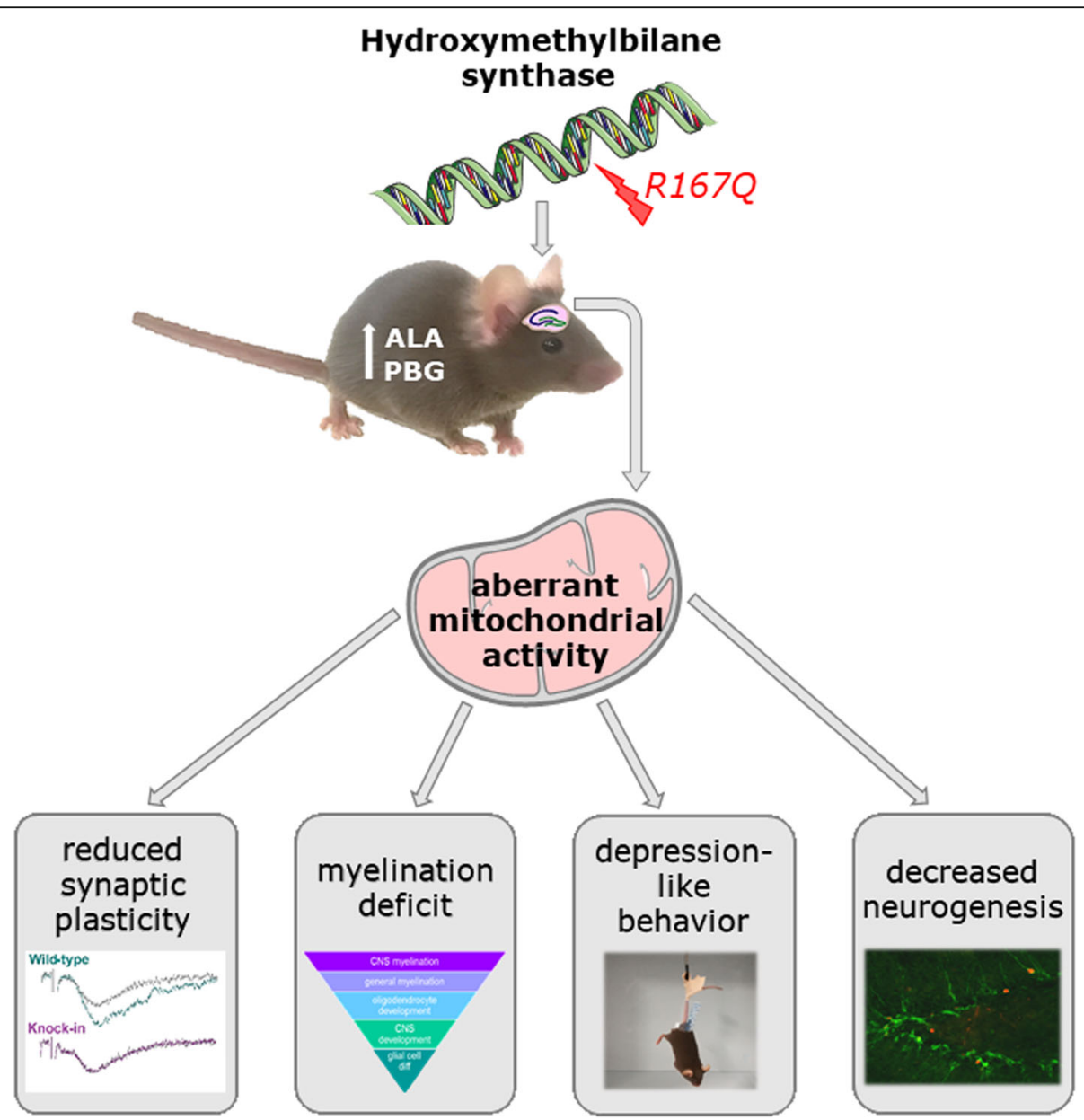

Fig. 7 Model for the pathomechanisms involved in augmented depression-like behavior in HMBS-deficient mice. Aberrant mitochondrial activity is proposed as central pathological feature impacting on neurogenic and neuroplastic potential, as well as on myelination in the KI hippocampus, all of which may collectively contributed to enhanced depression-like behavior in HMBS-deficient mice 
their CNS tissues. By extension, we propose that defective mitochondrial function may also contribute to the neuropsychiatric abnormalities that heterozygous AIP patients experience during acute attacks and should be considered for future development of preventive and therapeutic strategies for the clinical management of the disease.

\section{Conclusion}

This study for the first time reveals the behavioral consequences of severe HMBS deficiency in a mouse model of homozygous-dominant AIP and proposes mitochondrial dysfunction as relevant pathophysiological mechanism.

\section{Supplementary information}

Supplementary information accompanies this paper at https://doi.org/10. 1186/s40478-020-00910-z.

\section{Additional file 1: Table S1. Description of samples. Samples sizes} (with sex and genotype) are reported according to experimental procedure.

Additional file 2: Table S2. 228 differentially expressed transcripts were downregulated in HMBS-deficient mice. Columns are as follows: Gene short-name, locus, sample $1+2$, value $1+2$ (mean reads RPKM), fold change, $p$-value, $q$-value. Table S3. 92 differentially expressed transcripts were upregulated in HMBS-deficient mice. Columns are as follows: Gene short-name, locus, sample $1+2$, value $1+2$ (mean reads RPKM), fold change, $p$-value, $q$-value.

Additional file 3: Figure S1. Basal TMRM fluorescence in cultured hippocampal neurons and pharmacological intervention with ATP synthase inhibitor (Oligomycin), complex III inhibitor (Antimycin A) and protonophore FCCP in $\mathrm{KI}$ and WT mice; $n=5-9$ cells per genotype. TMRM, tetramethyl rhodamine ethyl ester, FCCP, carbonyl cyanide-4(trifluoromethoxy)phenylhydrazone.

Additional file 4: Figure S2. Neuropathohistological evaluation of hippocampal coronal sections stained with hematoxylin-eosin (H\&E), SMI31 (phosphorylated epitope in neurofilament) and SMI-32 (non-phosphorylated epitope in neurofilament) of HMBS-deficient (KI) and wildtype (WT) mice; $n=8-12$ per genotype.

\section{Abbreviations}

AIP: Acute Intermittent Porphyria; ALA: Aminolevulinc acid; ATP: Adenosinetriphosphat; CI-V: Complex I-V; CNP: 2',3'-Cyclic-nucleotide 3'phosphodiesterase; DAB: 3,3'-diaminobenzidine; DCX: Doublecortin; DG: Dentate gyrus; EPM: Elevated plus maze; FCCP: Carbonyl cyanide-ptrifluoromethoxyphenylhydrazone; FDR: False discovery rate; GFAP: Glial fibrillary acidic protein; HD: Homozygous dominant; HE: Hematoxylin-eosin; HMBS: Hydroxymethylbilane synthase; KI: Knock in; LD: Light dark; LTP: Longterm potentiation; MDD: Major depressive disorder; MOG: Myelin oligodendrocytic protein; MOM: Mouse on mouse; MPP: Medial perforant path; MS: Multiple sclerosis; NADH: Nicotinamide adenine dinucleotide hydrogen; NSF: Novelty suppressed feeding; PBG: Phorphobilinogen; PCR: Polymerase chain reaction; PFA: Paraformaldehyd; PLP: Proteolipid protein; PPI: Paired pulse inhibition; RL: Radioligand; RNA: Ribonucleic acid; SEM: Standard error of mean; SPT: Sucrose preference test; TMRM: Tetramethyl-rhodamine-methyl-ester; TST: Tail suspension test: WT: Wildtype

\section{Acknowledgements}

The authors thank Prof. Robert Eferl and Gerald Timelthaler, Msc for microscope image scanning and the Biomedical Sequencing Facility of the Center for Molecular Medicine of the Austrian Academy of Sciences for support of RNA-Seq experiments. Furthermore, the authors wish to express their gratitude to Dr. Christian Schubert for help with mitochondrial enzyme measurements, Maureen Cabatic, Msc and Orsolya Horvath, Msc for animal maintenance and genotyping and Irene Erber and Friederike Steudle, Msc for the help with immunohistochemical analysis.

\section{Authors' contributions}

Conceptualization: DDP, SB, SG-P, MY, RJD; Methodology: SB, MS, AC, FJM, PC, MH, GR, JH; Analysis and data interpretation: SB, MH, AC, FJM, JH, DDP; Writing manuscript: DDP, SB, MY, RJD; Supervision,: DDP; Funding acquisition: DDP, SB. The author(s) read and approved the final manuscript.

\section{Funding}

This project was supported by a stand-alone grant of the Austrian Science Fund (Fonds für Wissenschaft und Forschung, FWF; grant no. P30461) to DDP and a DOC fellowship of the Austrian Academy of Sciences to SB.

\section{Availability of data and materials}

The datasets used and analyzed during the current study are available from the corresponding author on reasonable request and raw data of differentially expressed genes are available at www.ncbi.n/m.nih.gov/geo/ query/acc.cgi?acc=GSE146212.

\section{Ethics approval and consent to participate}

All experiments concerning mice were carried out respecting the ARRIVE guidelines and the U.K. Animals (Scientific Procedures Act, 1986 and associated guidelines, EU Directive 2010/63/EU for animal experiments) and were approved by the national ethical committee on animal care and use (BMBWF66.009/0175-V/3b/2019- Bundesministerium für Wissenschaft und Forschung).

\section{Consent for publication}

Not applicable.

\section{Competing interests}

MY and RJD are past recipients of research grants from Alnylam Pharmaceuticals and Recordati Rare Diseases. They are co-inventors of a patent licensed to Alnylam Pharmaceuticals for RNAi therapy of the acute hepatic porphyrias. RJD is a consultant for Alnylam Pharmaceuticals, Recordati Rare Diseases, and Mitsubishi Tanabe Pharma Development America. The other authors declare no conflict of interest.

\section{Author details}

${ }^{1}$ Department of Neurophysiology and Neuropharmacology, Center of Physiology and Pharmacology, Medical University of Vienna, Schwarzspanierstrasse, 17, A-1090 Vienna, Austria. ²Department of Neurology, Division of Neuropathology and Neurochemistry, Medical University of Vienna, Vienna, Austria. ${ }^{3}$ Department of Pediatric and Adolescent Medicine, Medical University of Vienna, Vienna, Austria. ${ }^{4}$ Icahn School of Medicine at Mount Sinai, New York, NY, USA.

Received: 27 January 2020 Accepted: 2 March 2020

Published online: 20 March 2020

\section{References}

1. Puy H, Gouya L, Deybach JC (2010) Porphyrias. Lancet 375:924-937 https:// doi.org/10.1016/S0140-6736(09)61925-5

2. Balwani M, Desnick RJ (2012) The porphyrias: advances in diagnosis and treatment. Blood 120:4496-4504 https://doi.org/10.1182/blood-2012-05-423186

3. Narang N, Banerjee A, Kotwal J et al (2003) Psychiatric manifestations in three cases of acute intermittent porphyria. Med J Armed Forces India 59: 171-173 https://doi.org/10.1016/S0377-1237(03)80075-8

4. Crimlisk HL (1997) The little imitator--porphyria: a neuropsychiatric disorder. J Neurol Neurosurg Psychiatry 62:319-328

5. Burgovne K, Swartz R, Ananth J (1995) Porphyria: reexamination of psychiatric implications. Psychother Psychosom 64:121-130

6. González-Arriaza HL, Bostwick JM (2003) Acute Porphyrias: a case report and review. Am J Psychiatry 160:450-459 https://doi.org/10.1176/appi.ajp.160.3.450

7. Bonkovsky HL, Maddukuri VC, Yazici C et al (2014) Acute porphyrias in the USA: features of 108 subjects from porphyria consortium. Am J Med 127: 1233-1241 https://doi.org/10.1016/j.amjmed.2014.06.036

8. Bissell DM, Wang B (2015) Acute hepatic porphyria. J Clin Transl Hepatol 3 : 17-26 https://doi.org/10.14218/JCTH.2014.00039 
9. Handschin C, Lin J, Rhee J et al (2005) Nutritional regulation of hepatic heme biosynthesis and porphyria through PGC-1a. Cell 122:505-515 https:// doi.org/10.1016/j.cell.2005.06.040

10. Miller LK, Kappas A (1974) The effect of progesterone on activities of $\delta$ aminolevulinic acid synthetase and $\delta$-aminolevulinic acid dehydratase in estrogen-primed avian oviduct. Gen Comp Endocrinol 22:238-244 https:// doi.org/10.1016/0016-6480(74)90114-2

11. Granick S (1963) Induction of the synthesis of $\delta$-amino-levulinic acid synthetase in liver parenchyma cells in culture by chemicals that induce acute porphyria. J Biol Chem 238:PC2247-PC2249

12. Llewellyn DH, Smyth SJ, Elder GH et al (1992) Homozygous acute intermittent porphyria: compound heterozygosity for adjacent base transitions in the same codon of the porphobilinogen deaminase gene. Hum Genet 89:97-98 https://doi.org/10.1007/BF00207051

13. Yasuda M, Gan L, Chen B et al (2019) Homozygous hydroxymethylbilane synthase knock-in mice provide pathogenic insights into the severe neurological impairments present in human homozygous dominant acute intermittent porphyria. Hum Mol Genet 28:1755-1767 https://doi.org/10. 1093/hmg/ddz003

14. Solis C, Martinez-Bermejo A, Naidich TP et al (2004) Acute intermittent porphyria: studies of the severe homozygous dominant disease provides insights into the neurologic attacks in acute porphyrias. Arch Neurol 61: 1764-1770 https://doi.org/10.1001/archneur.61.11.1764

15. Lindberg RL, Porcher C, Grandchamp B et al (1996) Porphobilinogen deaminase deficiency in mice causes a neuropathy resembling that of human hepatic porphyria. Nat Genet 12:195-199 https://doi.org/10.1038/ng0296-195

16. Yasuda M, Gan L, Chen B et al (2014) RNAi-mediated silencing of hepatic Alas1 effectively prevents and treats the induced acute attacks in acute intermittent porphyria mice. Proc Natl Acad Sci 111:7777-7782 https://doi. org/10.1073/pnas.1406228111

17. Yasuda M, Bishop DF, Fowkes M et al (2010) AAV8-mediated gene therapy prevents induced biochemical attacks of acute intermittent porphyria and improves neuromotor function. Mol Ther 18:17-22 https:/doi.org/10.1038/mt.2009.250

18. Lindberg RL, Martini R, Baumgartner M et al (1999) Motor neuropathy in porphobilinogen deaminase-deficient mice imitates the peripheral neuropathy of human acute porphyria. J Clin Invest 103:1127-1134 https:/doi.org/10.1172/JCI5986

19. Pollak DD, Monje FJ, Zuckerman $L$ et al (2008) An animal model of a behavioral intervention for depression. Neuron 60:149-161

20. Monje FJ, Cabatic M, Divisch I et al (2011) Constant darkness induces IL-6dependent depression-like behavior through the NF-kB signaling pathway. J Neurosci 31:9075-9083

21. Savalli G, Diao W, Schulz S et al (2015) Diurnal oscillation of amygdala clock gene expression and loss of synchrony in a mouse model of depression. Int J Neuropsychopharmacol 18 https://doi.org/10.1093/ijnp/pyu095

22. Britton DR, Britton KT (1981) A sensitive open field measure of anxiolytic drug activity. Pharmacol Biochem Behav 15:577-582 https://doi.org/10.1016/ 0091-3057(81)90212-4

23. Mineur YS, Picciotto MR, Sanacora G (2007) Antidepressant-like effects of ceftriaxone in male C57BL/6J mice. Biol Psychiatry 61:250-252 https://doi. org/10.1016/j.biopsych.2006.04.037

24. Khan D, Fernando P, Cicvaric A et al (2014) Long-term effects of maternal immune activation on depression-like behavior in the mouse. Transl Psychiatry 4:e363

25. Can A, Dao DT, Terrillion CE et al (2012) The tail suspension test. J Vis Exp: e3769-e3769 https://doi.org/10.3791/3769

26. Kong E, Sucic S, Monje FJ et al (2015) STAT3 controls IL6-dependent regulation of serotonin transporter function and depression-like behavior. Sci Rep 5 https://doi.org/10.1038/srep09009

27. Franklin KBJ, Paxinos G (2013) Paxinos and Franklin's the mouse brain in stereotaxic coordinates

28. Meshi D, Drew MR, Saxe M et al (2006) Hippocampal neurogenesis is not required for behavioral effects of environmental enrichment. Nat Neurosci 9: 729-731 https://doi.org/10.1038/nn1696

29. Ramirez-Amaya V, Marrone DF, Gage FH et al (2006) Integration of new neurons into functional neural networks. J Neurosci 26:12237-12241 https:// doi.org/10.1523/JNEUROSCI.2195-06.2006

30. Gould E, Cameron HA, Daniels DC et al (1992) Adrenal hormones suppress cell division in the adult rat dentate gyrus. J Neurosci 12:3642-3650 https:// doi.org/10.1523/JNEUROSCI.12-09-03642.1992

31. Plumpe T, Ehninger D, Steiner B et al (2006) Variability of doublecortinassociated dendrite maturation in adult hippocampal neurogenesis is independent of the regulation of precursor cell proliferation. BMC Neurosci 7:77 https://doi.org/10.1186/1471-2202-7-77

32. Cicvaric A, Yang J, Krieger S et al (2016) The brain-tumor related protein podoplanin regulates synaptic plasticity and hippocampus-dependent learning and memory. Ann Med 48:652-668 https:/doi.org/10.1080/07853890.2016.1219455

33. Kim D, Pertea G, Trapnell C et al (2013) TopHat2: accurate alignment of transcriptomes in the presence of insertions, deletions and gene fusions. Genome Biol 14:R36 https://doi.org/10.1186/gb-2013-14-4-r36

34. Trapnell C, Hendrickson DG, Sauvageau M et al (2013) Differential analysis of gene regulation at transcript resolution with RNA-seq. Nat Biotechnol 31: 46-53 https://doi.org/10.1038/nbt.2450

35. Huang da W, Sherman BT, Lempicki RA (2009) Systematic and integrative analysis of large gene lists using DAVID bioinformatics resources. Nat Protoc 4:44-57 https://doi.org/10.1038/nprot.2008.211

36. Huang da W, Sherman BT, Lempicki RA (2009) Bioinformatics enrichment tools: paths toward the comprehensive functional analysis of large gene lists. Nucleic Acids Res 37:1-13 https://doi.org/10.1093/nar/gkn923

37. Chen EY, Tan CM, Kou Y et al (2013) Enrichr: interactive and collaborative HTML5 gene list enrichment analysis tool. BMC Bioinformatics 14:128 https://doi.org/10.1186/1471-2105-14-128

38. Kuleshov MV, Jones MR, Rouillard AD et al (2016) Enrichr: a comprehensive gene set enrichment analysis web server 2016 update. Nucleic Acids Res 44: W90-W97 https://doi.org/10.1093/nar/gkw377

39. Bankhead P, Loughrey MB, Fernández JA et al (2017) QuPath: open source software for digital pathology image analysis. Sci Rep 7:1-7 https://doi.org/ 10.1038/s41598-017-17204-5

40. Spinazzi M, Casarin A, Pertegato $V$ et al (2012) Assessment of mitochondrial respiratory chain enzymatic activities on tissues and cultured cells. Nat Protoc 7:1235-1246 https://doi.org/10.1038/nprot.2012.058

41. Geist B, Vorwerk B, Coiro P et al (2012) PRG-1 transcriptional regulation independent from Nex1/Math2-mediated activation. Cell Mol Life Sci 69: 651-661 https://doi.org/10.1007/s00018-011-0774-7

42. Rottenberg H, Wu S (1998) Quantitative assay by flow cytometry of the mitochondrial membrane potential in intact cells. Biochim Biophys Acta BBA - Mol Cell Res 1404:393-404 https://doi.org/10.1016/S01674889(98)00088-3

43. Schindelin J, Arganda-Carreras I, Frise E et al (2012) Fiji: an open-source platform for biological-image analysis. Nat Methods 9:676-682 https://doi. org/10.1038/nmeth.2019

44. Berger S, Ronovsky M, Horvath $\mathrm{O}$ et al (2018) Impact of maternal immune activation on maternal care behavior, offspring emotionality and intergenerational transmission in C3H/He mice. Brain Behav Immun 70:131-140

45. Ronovsky M, Berger S, Zambon A et al (2017) Maternal immune activation transgenerationally modulates maternal care and offspring depression-like behavior. Brain Behav Immun 63:127-136 https://doi.org/10.1016/j.bbi.2016.10.016

46. Masi G, Brovedani P (2011) The hippocampus, neurotrophic factors and depression: possible implications for the pharmacotherapy of depression. CNS Drugs 25:913-931 https://doi.org/10.2165/11595900-000000000-00000

47. Krishnan V, Nestler EJ (2008) The molecular neurobiology of depression. Nature 455:894-902

48. Nestler EJ, Barrot M, DiLeone RJ et al (2002) Neurobiology of depression. Neuron 34:13-25 https://doi.org/10.1016/S0896-6273(02)00653-0

49. Pinero J, Queralt-Rosinach N, Bravo A et al (2015) DisGeNET: a discovery platform for the dynamical exploration of human diseases and their genes. Database Oxf 2015:bav028 https://doi.org/10.1093/database/bav028

50. Homedan C, Laafi J, Schmitt C et al (2014) Acute intermittent porphyria causes hepatic mitochondrial energetic failure in a mouse model. Int J Biochem Cell Biol 51:93-101 https://doi.org/10.1016/j.biocel.2014.03.032

51. Homedan C, Schmitt C, Laafi J et al (2015) Mitochondrial energetic defects in muscle and brain of a Hmbs-/- mouse model of acute intermittent porphyria. Hum Mol Genet 24:5015-5023 https://doi.org/10.1093/hmg/ddv222

52. Scaduto RC, Grotyohann LW (1999) Measurement of mitochondrial membrane potential using fluorescent rhodamine derivatives. Biophys J 76:469-477

53. Griesauer I, Diao W, Ronovsky M et al (2014) Circadian abnormalities in a mouse model of high trait anxiety and depression. Ann Med 46:148-154 https://doi.org/10.3109/07853890.2013.866440

54. Sacchet MD, Gotlib IH (2017) Myelination of the brain in major depressive disorder: an in vivo quantitative magnetic resonance imaging study. Sci Rep 7:2200 https://doi.org/10.1038/s41598-017-02062-y

55. Liu J, Dupree JL, Gacias M et al (2016) Clemastine enhances myelination in the prefrontal cortex and rescues behavioral changes in socially isolated 
mice. J Neurosci 36:957-962 https://doi.org/10.1523/JNEUROSCl.3608-15. 2016

56. Pepper RE, Pitman KA, Cullen CL, Young KM (2018) How do cells of the oligodendrocyte lineage affect neuronal circuits to influence motor function, memory and mood? Front Cell Neurosci 12:399 https://doi.org/10. 3389/fncel.2018.00399

57. Gibson JB, Goldberg A (1956) The neuropathology of acute porphyria. J Pathol Bacteriol 71:495-509

58. Bansal Y, Kuhad A (2016) Mitochondrial dysfunction in depression. Curr Neuropharmacol 14:610-618 https://doi.org/10.2174/ 1570159X14666160229114755

59. Allen J, Romay-Tallon R, Brymer KJ et al (2018) Mitochondria and mood: mitochondrial dysfunction as a key player in the manifestation of depression. Front Neurosci 12 https://doi.org/10.3389/fnins.2018.00386

60. Kim HJ, Khalimonchuk O, Smith PM, Winge DR (2012) Structure, function, and assembly of heme centers in mitochondrial respiratory complexes. Biochim Biophys Acta BBA - Mol Cell Res 1823:1604-1616 https://doi.org/10. 1016/j.bbamcr.2012.04.008

61. Chinopoulos C, Adam-Vizi V (2010) Mitochondria as ATP consumers in cellular pathology. Biochim Biophys Acta (BBA) - Mol Basis Dis 1802:221-227 https://doi.org/10.1016/j.bbadis.2009.08.008

62. Campbell S, MacQueen G (2004) The role of the hippocampus in the pathophysiology of major depression. J Psychiatry Neurosci 29:417-426

63. Khacho M, Harris R, Slack RS (2019) Mitochondria as central regulators of neural stem cell fate and cognitive function. Nat Rev Neurosci 20:34-48 https://doi.org/10.1038/s41583-018-0091-3

64. Todorova V, Blokland A (2017) Mitochondria and synaptic plasticity in the mature and aging nervous system. Curr Neuropharmacol 15:166-173

65. Zhang Z, Wang W, Zhong P et al (2015) Blockade of 2-arachidonoylglycerol hydrolysis produces antidepressant-like effects and enhances adult hippocampal neurogenesis and synaptic plasticity. Hippocampus 25:16-26 https://doi.org/10.1002/hipo.22344

66. Vose LR, Stanton PK (2017) Synaptic plasticity, metaplasticity and depression. Curr Neuropharmacol 15:71-86 https://doi.org/10.2174/ 1570159X14666160202121111

67. Mahad D, Lassmann H, Turnbull D (2008) Review: mitochondria and disease progression in multiple sclerosis. Neuropathol Appl Neurobiol 34:577-589 https://doi.org/10.1111/j.1365-2990.2008.00987.x

68. Andrews HE, Nichols PP, Bates D, Turnbull DM (2005) Mitochondrial dysfunction plays a key role in progressive axonal loss in multiple sclerosis. Med Hypotheses 64:669-677 https://doi.org/10.1016/j.mehy.2004.09.001

\section{Publisher's Note}

Springer Nature remains neutral with regard to jurisdictional claims in published maps and institutional affiliations.

Ready to submit your research? Choose BMC and benefit from:

- fast, convenient online submission

- thorough peer review by experienced researchers in your field

- rapid publication on acceptance

- support for research data, including large and complex data types

- gold Open Access which fosters wider collaboration and increased citations

- maximum visibility for your research: over $100 \mathrm{M}$ website views per year

At BMC, research is always in progress.

Learn more biomedcentral.com/submissions 